\title{
Entre Georges et Claude Place des Géorgiques dans l'œuvre de Claude Simon
}

\section{Aymeric Glacet}

\section{(2) OpenEdition}

1 Journals

Édition électronique

URL : http://journals.openedition.org/ccs/861

DOI : 10.4000/ccs.861

ISSN : 2558-782X

Éditeur :

Presses universitaires de Rennes, Association des lecteurs de Claude Simon

\section{Édition imprimée}

Date de publication : 31 mars 2013

Pagination : 83-99

ISBN : 9782354121785

ISSN : $1774-9425$

\section{Référence électronique}

Aymeric Glacet, «Entre Georges et Claude Place des Géorgiques dans l'œuvre de Claude Simon », Cahiers Claude Simon [En ligne], 8| 2013, mis en ligne le 21 septembre 2017, consulté le 28 avril 2019. URL : http://journals.openedition.org/ccs/861 ; DOI : 10.4000/ccs.861 


\title{
Entre Georges et Claude Place des Géorgiques dans l'œuvre de Claude Simon
}

\author{
Aymeric GLACET \\ Sewanee, The University of the South, USA
}

Il n’y a que les gens médiocres pour penser à tout.

Pierre Grassou, Honoré de Balzac

Les Géorgiques'. Et pourquoi pas Les Georges, tout simplement. $\mathrm{Ou}$ " la geste de Georges ${ }^{2}$ ", pour reprendre l'expression de Lucien Dällenbach. Georges, fils d'Henri ; Georges, amant de Corinne ; Georges, mari de Louise ; Georges, orphelin élevé par ses deux vieilles tantes Marie et Eugénie ; ou Georges encore, dans L'Acacia et dans Les Géorgiques, narrateur d'autant plus présent qu'il n'est pas nommé alors qu'il est quasi autobiographique.

Quasi, en effet, ce que confirme Alastair Duncan lorsqu'il explique que dans ce roman, "malgré tout ce qui appartient au do-

1. Claude Simon, Les Géorgiques, Minuit, Paris, 1981. Abréviation G.

2. Lucien Dällenbach, Claude Simon, Seuil, "Les Contemporains ", 1988, note 97, p. 210. 
maine du fictif, Simon est enfin arrivé à proposer sa propre famille comme base de toute invention ${ }^{3}$ "; Simon qui répète à qui veut l'entendre, dans l'esprit de Montaigne pour qui le magasin de la mémoire est volontiers plus fourni de matière que n'est celui de l'invention : "Je suis incapable d'inventer quoi que ce soit ${ }^{4}$ ". N'étant capable d'inventer, en conséquence, que ce qu'il a vécu... Un vécu qui, parce qu'il est, somme toute, comme il l'explique en 1988 dans une notice autobiographique, assez modeste - deux pages qui se résument à la paresse, l'oisiveté, l'ennui, le sport, un peu de lecture, quinze jours à Barcelone, une retraite sur la route des Flandres, un séjour à l'hôpital -, est aussi, à partir des Géorgiques, artificiellement ${ }^{5}$ dopé à l'histoire de famille sous prétexte que cette histoire-là, après tout, elle ne s'invente pas. Qu'importe alors si l'écrivain ne l'a pas vécue en personne, il est le mieux placé pour la vivre par procuration, ce que l'oncle Charles, rite de passage oblige, lui explique le jour où il met à sa disposition les archives familiales : "Peut-être es-tu encore trop jeune, mais plus tard... Quand tu seras vieux toi-même. Je veux dire quand tu seras capable non pas de comprendre mais de sentir les choses parce que tu les auras toi-même éprouvées... " (Les Géorgiques, p. 445) Claude Simon, comme un bon petit soldat, ne s'allant faire la guerre, rétrospectivement, que pour être un jour à la hauteur de la biographie familiale dont il est le seul dépositaire : "En tout cas vous avez quelque chose en commun, ajoutait l'oncle Charles à propos de Jean-Pierre Lacombe Saint-Michel : tu as fait toi-même la guerre sur un cheval» (G, p. 446).

L'un comme l'autre, en effet, l'ancêtre et l'écrivain, ont entendu les fers des chevaux marteler ( $G$, p. 41), crépiter $(G$, p. 49) sur le pavé lorsqu'il ne fallait pas "se battre sans bêtes, sans vivres et sans armes " ( $G$, p. 244). Mais à la différence de Claude Simon, le patriarche monumental qui participa à la prise de la Bastille et au procès de Louis

3. Alastair Duncan, "Claude Simon : le projet autobiographique ", in Claude Simon, Revue des Sciences Humaines, 220, 1990-4, p. 60.

4. Madeleine Chapsal, "Entretien avec Claude Simon ", in L'Express, 10 novembre 1960. Repris dans Madeleine Chapsal, Les Ecrivains en personne, pp. 163171.

5. Voir le sens donné à ce mot par Claude Simon dans son Discours de Stockholm. 
XVI, ce « J.P.L.S.M. Député du Tarn» ( $G$, p. 180) qui vota contre l'appel au peuple, pour la mort et contre le sursis, l'" arrière-grandpère " $(G$, p. 255) colossal et statufié qui évita de justesse le sort de son ami Danton, était une armée à lui tout seul qui conduisait des assauts « simplement d'un chapeau à plumes, d'une écharpe et d'une épée » $(G$, p. 394).

Autant dire que Claude Simon l'admire, cet érudit Minotaure qui a fait "la plus grande révolution de l'Histoire» (G, p. 222), ce "paysan» $(G$, p. 447$)$ cultivé pour qui il n'y avait aucun " espoir d'acquérir de la gloire» lorsqu'il n'y avait "pas un coup de fusil de tiré» $(G$, p. 473), l'infirme boiteux qui a fait, selon les sources, de dix-sept à vingt-deux campagnes, a été blessé de deux à quatre fois, et puis après quarante-sept ans de service sans interruption, a pris sa retraite avant d'être terrassé par une attaque d'apoplexie à l'âge de cinquante-neuf ans, une canne à la main. Claude Simon l'admire, ce grand officier de la Légion d'honneur, chevalier de la Couronne de Fer, membre de l'Académie de Milan, et compare, autant que faire se peut, sa carrière à la sienne, s'imaginant peut-être qu'elle aurait été toute différente s'il avait servi sous ses ordres plutôt que de vivre à propos.

Mais ce qui me paraît plus intéressant encore dans cette comparaison, c'est que Claude Simon a choisi de ne pas inclure Les Géorgiques dans le premier volume de ses œuvres destinées à la $\mathrm{Bi}$ bliothèque de la Pléiade alors même que ce roman est le seul qu'il mentionne dans son Discours de Stockholm, et qu'il le mentionne pour cette raison qu'il se targue, quelques lignes plus loin, de pouvoir ranger son œuvre, qui n'avait d'autre ambition que de faire de «l'art pour l'art ", " parmi les instruments d'une action révolutionnaire et déstabilisatrice " obligeant même, suprême honneur, le K.G.B. soviétique à noyauter l'Académie suédoise. De la Révolution française à l'Union soviétique comme «symbole de redoutables forces de subversion auxquelles il est [...] flatteur pour un simple écrivain de se voir associé6 ", c'est à ce niveau-là, qui est un niveau éminemment symbolique évidemment, que la comparaison se joue entre L.S.M. et Claude Simon.

6. Claude Simon, Euvres, «Bibliothèque de la Pléiade ", Gallimard, Paris, 2006, p. 889. 
De là à ce que l'écrivain conçoive aussi aisément Socrate en la place d'Alexandre, qu'Alexandre en celle de Socrate, je ne puis l'affirmer. Mais si Alastair Duncan voit peu à peu Claude Simon devenir la matière de son ouvre, c'est parce que l'autobiographie des uns est toujours, tout compte fait, l'affaire de famille des autres : "Simon récupère son œuvre, explique-t-il ; rétrospectivement, il la transforme en autobiographie; il s'engage à avoir parlé de lui - dans la mesure du possible. " Dans la mesure du possible, en effet, si l'on considère que la " disparition progressive du fictif ${ }^{8}$ " se fait au profit du biographique, et que l'écrivain parle de lui pourvu qu'il parle de Lacombe Saint-Michel. "Profondément d'accord avec la phrase de Proust qui dit que la réalité ne se forme que dans le souvenir" ", c'est pour autant alors que la mémoire des siens puisse aussi être la sienne. Et si le roman Les Géorgiques est une œuvre de synthèse ${ }^{10}$, c'est à cette seule condition que la mémoire, comme une montre, se lègue de père en fils.

Pas d'autre réalité, autrement dit, que celle de Georges, Georges et ses multiples apparitions, et qui, à chaque nouvelle apparition, synchronise les horloges, s’assure que le temps de référence est pour toutes le même, et accommode l'histoire à l'heure, Georges le principal témoin de l'œuvre qui assiste à la mort de de Reixach sur la route des Flandres le 17 mai 1940 sur le coup de cinq heures du soir ${ }^{11}$; pas d'autre réalité que celle-là du moment où cette fiction, qui est créée "à partir de pièces historiques ${ }^{12}$ ", s'élabore " de plus en plus près de l'auteur ${ }^{13}$ " : « [J]'ai retenu assez d'histoires de famille, de ma

7. Alastair Duncan, "Claude Simon : le projet autobiographique ", in Claude Simon, Revue des Sciences Humaines, 220, 1990-4, p. 60.

8. Marianne Alphant, " "Et à quoi bon inventer” ", Libération, 31 août 1989.

9. Madeleine Chapsal, «Entretien, Claude Simon parle ", L'Express, 5 avril 1962, p. 32 .

10. Claude Simon, Euvres, «Bibliothèque de la Pléiade », Gallimard, Paris, 2006, p. XL.

11. Voir Aymeric Glacet, Claude Simon Chronophotographe ou les Onomatopées du temps, Septentrion, collection «Claude Simon », 2007, p. 151-195.

12. Claude Simon, Euvres, op. cit., p. XLIV.

13. Claude Simon, Euvres, ibid., p. XLIV. 
famille, pour emplir longtemps mes livres ${ }^{14}$ ", disait Claude Simon en 1961. Les Géorgiques, vingt ans plus tard, ressemble fort à un roman familial, et Georges, d'une réincarnation à l'autre, n'a jamais été aussi proche de Claude.

Reste que pour parvenir à ses fins, pour aligner les destins comme s'alignent les étoiles, le mobile doit repasser successivement par les mêmes points. Et Georges, pour que la révolution soit complète, doit repasser par Lacombe-Saint-Michel. Convaincu que "plus on écrit, plus on a de souvenirs ${ }^{15}$ ", Claude Simon s'approprie définitivement son aïeul. C'est que plus on est de fous, plus on a de mémoire... L'œuvre, qui en consomme en grande quantité, élargit le cercle familial et recycle alors, une fois épuisées les cartes postales du père et les photographies de la mère, la mémoire des anciens. Mais contrairement à ce que lui enseignait l'oncle Charles, ce n'est pas en sentant les choses qu'il y parviendra : "Il me semble, écrit-il, que le livre se fait au niveau de l'écriture, du langage ${ }^{16}$. " Citant à plusieurs reprises le mot de Raoul Dufy, et abandonnant ce qu'il a voulu faire au profit de ce qui se fait, il constate dès 1969 : « Je disais autrefois : il est possible de reconstituer à partir de choses vécues, senties. Aujourd'hui, après avoir réfléchi, je ne pense plus qu'on puisse « reconstituer " quoi que ce soit. Ce que l'on constitue, c'est un texte, et ce texte ne correspond qu'à une seule chose : à ce qui se passe en l'écrivain au moment où il l'écrit ${ }^{17}$. » Ce qui arrive, ce qui se fait, ce qui se passe, définition de l'écriture par Claude Simon. Car n'est vécue que la page d'écriture. La sienne, évidemment. Mais celle aussi, désormais, de L.S.M.

14. Entretien avec Denise Bourdet, "Images de Paris : Claude Simon ", La revue de Paris, $\mathrm{n}^{\circ}$ 68, janvier 1961, p. 139 ; repris dans D. Bourdet, Brèves rencontres, Grasset, 1963.

15. "L'inlassable réa/encrage du vécu ", entretien avec Mireille Calle-Gruber, Claude Simon. Chemins de la mémoire, p. 18.

16. André Bourin, "Le Roman jugé par Nathalie Sarraute, M. de Saint Pierre, Claude Simon, Jean Hougron, J.-R. Huguenin ", Les Nouvelles littéraires, 22 juin 1961, p. 7.

17. Bettina L. Knapp, "Interview avec Claude Simon », Kentucky Romance Quaterly (Lexington), vol. 17, $\mathrm{n}^{\circ}$ 2, 1970, p. 182. 
Comme si les archives de ce dernier, tout compte fait, étaient les brouillons de l'écrivain, et que ces brouillons, auxquels ils ne manquaient que de s'inscrire dans le roman simonien, d'y trouver leur place depuis toujours réservée, étaient plus à même de constituer le vécu de Claude Simon que de reconstituer celui de son ascendant. Le grand récit n'est jamais qu'une combinaison de petits récits, Claude Simon nous le rappelle sans cesse. Et souvent avec ironie, tant ces petits récits sont des fragments de la grande Histoire, les restes de l'histoire révolutionnaire, les bagatelles de l'histoire napoléonienne, alors qu'il s'agit tout de même de l'histoire, mais de " roman » cette fois, d'un homme "à la fois impulsif et réfléchi, capable de passer brusquement de la réflexion à l'action, du silence à l'apostrophe " $(G$, p. 55), de l'histoire, la seule qui compte véritablement pour l'écrivain, d'un lion qui s'est battu comme dix, de l'histoire, par conséquent, dont on pouvait croire que Claude Simon l'admirait alors qu'il ne faisait que la vivre.

Considérant que la vie elle-même est un brouillon où le héros, comme la phrase parfaite et exemplaire, ne peut être que le héros d'un jour, d'une heure ou d'un instant, le reste du temps se passe, sabre au mur, à tâtonner, griffonner, raturer, corriger ce qui a été écrit la veille, fût-ce par un aïeul. Ainsi le vécu simonien va-t-il brouillant les vies entre elles comme s'embrouillent les phrases afin que se retrouvent assis l'un à côté de l'autre, inévitable anachronisme du présent de l'écriture, Georges et L.S.M. Ce n'est plus seulement un même sang alors, mais une même encre boueuse qui coule dans leurs veines. Si «c'est seulement en écrivant que quelque chose se produit » (Préface à Orion aveugle), encore faut-il que l'écriture prenne racine. Pas de meilleur terreau que cette boue couleur rouille des Géorgiques! Georges, celui qui travaille la terre, capable de distinguer le seigle du blé, ou le peuplier du tremble, est celui par qui l'œuvre se ressource. Bien qu'il soit toujours un personnage de premier plan dans les romans simoniens, il est cette fois « issu [...] de la terre ellemême » ( $G$, p. 287), c'est-à-dire non pas de n'importe quelle terre, mais de la terre que L.S.M. retournait déjà comme on retourne "le monde pour ainsi dire [...] à la façon d'un gant, d'un vêtement, révélé dans son envers" $(G, \mathrm{p} .426)$. "Mois après mois, saison après saison " (Gulliver, p. 447), c'est l'envers de l'œuvre qui était ainsi révélée. 
Et d'ailleurs, à y regarder d'un peu plus près, les titres des romans simoniens peuvent se lire comme la preuve ultime de cet enracinement : en 1960, le titre La Route des Flandres fait référence à l'histoire contemporaine; en 1967, Histoire dépasse les seules limites de la Seconde Guerre mondiale ; en 1969, La Bataille de Pharsale devient la guerre à l'origine de toutes les guerres et l'histoire à l'origine de toutes les histoires ; et finalement, en 1981, le titre Les Géorgiques renvoie à l'origine tellurique de l'humanité, à cette terre retournée par le socle des armées, engraissée par les corps des soldats, édifiée par Virgile, entretenue par L.S.M. et sauvegardée par Georges qui remonte ainsi le temps au fur et à mesure que les titres, quant à eux, pour mieux faire remonter les corps, pour mieux révéler l'envers du décor, pour mieux retourner l'histoire, toutes les histoires, s'enfoncent dans la terre.

De la page blanche à la page arable, tout dépend donc de la façon dont elle est travaillée pour faire non pas que chaque mot remonte à la surface, mais qu'à la surface chaque mot ait son carrefour qui indique, comme une croix, le lieu où il est enterré. Découvrant que les mots les plus enfouis sont ceux qui ont le plus vécu, Claude Simon a beau constater, dans Histoire, leur impuissance à raconter l'expérience du feu pour l'" éprouver de nouveau après coup " (Histoire, p. 152), il sait aussi qu'il ne dispose "que de mots" (Histoire, p. 152), et que ces mots, puisqu'il est évident dans cette œuvre que l'arbre de Porphyre fonctionne aussi en rhizome, sont des lieux de circulation $^{18}$. Ainsi faut-il en prendre son parti, ce dont convenait le général d'Empire qui, entre deux rapports, deux discours ou deux projets d'armées, " annotait, corrigeait, embellissait, labourait, plantait par procuration, usant non de charrues ou de herses mais de cette encre brune, couleur rouille, sur le papier grenu des innombrables lettres envoyées à Batti » $(G, \mathrm{p} .377)$.

Pour lui qui voulait, comme Montaigne, que la mort le trouve plantant des choux, travailler signifiait " travailler et fumer le pied des treilles ", " travailler les acacias" ou " travailler les pépinières"

18. Voir Préface d'Orion aveugle et "La Fiction mot à mot ", in Claude Simon, Euvres, "Bibliothèque de la Pléiade ", Gallimard, Paris, 2006, Appendices, p. 1189-1190. 
( $G$, p. 475). "La main à plume vaut la main à charrue ", affirmait Rimbaud. Dans le cas de L.S.M., c'était d'autant plus vrai qu'il passa sa vie à "labour[er] et moissonn[er] les champs en esprit " ( $G$, p. 366). Travailler la terre en esprit revenant, en littérature, et pour Claude Simon cette fois, à travailler, faute de mot pour le dire autrement, " au hasard de l'inspiration ${ }^{19}$ ", tout comme c'est au " hasard de la chance " $(G$, p. 82) que seront mises à jour les archives familiales, paperasses, registres, lettres, instructions, ordres de marche, discours, contre-ordres et autres documents retrouvés dans un placard caché dont la porte ne sera découverte que lorsque la tapisserie viendra à manquer, ce placard qui est à la famille de Claude Simon ce que l' " armoire de fer " est à celle de Louis XVI, une armoire qui précipitera sa mise à mort votée par Lacombe Saint-Michel qui, à Paris, vivait « rue du Hasard au nom choisi, semblait-il, par le destin lui-même» $(G$, p. 399).

Et pour cause ! Précédemment rue du Hasard, pour une partie de la voie, et rue Thérèse, pour l'autre : ce n'est que le 23 octobre 1880 qu'est réunie la rue du Hasard à la rue Thérèse. La partie rue du Hasard existait pourtant dès 1622. Jusqu'en 1692, les deux parties étaient même confondues sous le nom de rue du Hasard. Il fallut attendre de Louis XIV qu'il épousât Marie-Thérèse d'Autriche pour qu'une moitié de la rue s'ennoblisse. Avant cela, il n'était question que du jeu, jeu de paume ou de jeu de quilles; on disait alors d'une balle qui rebondissait d'une façon insolite, ou d'une boule qu'un obstacle imprévu dérangeait une fois lancée, que cette balle ou cette boule faisait hasard. Mais surtout, comme synonyme d'as à l'usage des joueurs de dés, le hasard en était venu à servir de dénomination à un genre de partie. Une maison de jeu, par extension, gardait le nom du Hazard et le faisait partager à la ruelle où elle était située. Et c'est dans ce coupe-gorge du Hasard que s'engagea L.S.M. lorsqu'il se remaria avec une aristocrate et la logea, à deux pas du tripot, rue Marie-Thérèse d'Autriche ${ }^{20}$, une aristocrate pour laquelle, "se grattant un instant la tête, prenant au hasard un

19. Entretien avec Denise Bourdet, art. cit., p. 137.

20. D'après Charles Lefeuve, Histoire de Paris rue par rue, maison par maison, 1875. 
autre nom et le mettant à la place de celui qu'il venait de rayer » $(G$, p. 388), il avait, sur un coup de dés, " tout laissé, [et] tout risqué " ( $G$, p. 383).

Tricher, corriger le hasard - peut-on lire en exergue au Tricheur. Cette définition du Littré est la phrase d'ouverture de l'œuvre. Quelque chose comme sa préface, son avis au lecteur. Il n'y en pas d'autre. Aucune qui corresponde mieux à ce que Claude Simon cherche de nouveau à dire dans Les Géorgiques, lui qui veut nous faire accepter l'idée qu’il écrit comme on pipe des dés ou comme, sans jamais y mettre les pieds, L.S.M. cultive son jardin. Par correspondances, dirait Baudelaire. Mais si le roman Les Géorgiques est à la charnière de l'œuvre, ce n'est pas seulement parce que la plume est une herse, c'est aussi parce que le travail de l'écrivain n'est dû au hasard que lorsque le hasard se casse les dents sur les mots. Contre « le hasard, presque une question d'heure ", qui intervient pour qu'on se retrouve "un jour précis, à l'endroit précis et à l'heure précise » $(G, \mathrm{p}$. 354-355) qui font qu'à ce moment le destin bascule, contre ce hasard qui fait si bien les choses que « tout est combiné, machiné, arrangé de manière que de toute façon et n'importe comment qu'on s'y prenne on sera refait, roulé, comme si tout était minuté, fixé à l'avance, et le moment, et le rôle de chacun " (Le Sacre, p. 244), contre ces romans, écrit-il encore dans Le Sacre du printemps, " où on commence par vous présenter un tas de personnages sans lien apparent et qui vont tous se rencontrer sur le coup de l'heure la plus favorable à l'auteur comme à un rendez-vous en vue duquel ils ont été préparés et entraînés avec soin, suivant un horaire méticuleusement étudié ", Claude Simon réagit en critiquant cette trop gracieuse mécanique romanesque, "espèce de mécanisme où aucun rouage ne peut bouger sans que tous les autres se mettent aussitôt en branle " (Le Sacre, p. 244). Et le cas exemplaire que constitue son ancêtre qui convole rue du Hasard après avoir sauvé la tête d'une royaliste alors même qu'il ne lèvera pas le plus petit doigt pour sauver celle de son propre frère révèle combien "la réalité dépasse la fiction ${ }^{21}$ " et exige de l'écrivain qu'il soit, ô combien! à la hauteur des événements. Contre le roman traditionnel, contre le roman balzacien et la fonction diégétique de

21. Claude Simon, Euvres, op. cit., p. XLIV. 
la description, comme si le lieu pouvait expliquer la personne, et la personne, dans ses traits physiques, afficher son caractère, Simon, tout comme Balzac qui n'était pas complètement dupe de l'engrenage, sait qu'un plan, quelles que soient sa sagesse et son exécution, manque parfois "par un jeu du Hasard qui modifie tout ici-bas ${ }^{22}$. "

Et comme si c'était également le hasard, tout bien considéré, qui ferait que parlant de Lacombe Saint-Michel, Claude Simon ne parlerait en fait que de Balzac. Ou de son père, Bernard-François Balzac, issu lui aussi d'une famille de laboureurs, et qui n'aura d'autre protecteur, sous la Révolution, que son compatriote et ami tarnais, Jean-Pierre. Les Lacombe de Saint-Michel, originaires du bourg de Penne, dans la basse vallée de l'Aveyron, étaient à l'origine des artisans cordiers avant de devenir, au début du XVII siècle, marchands et de figurer parmi les plus riches familles de la bourgade. Ce fut Eugène Lacombe (1655-1735), premier du nom, qui devint maire de la communauté de Penne en 1693 et hérita par son mariage de la coseigneurie de Saint-Michel-de-Vax qu'il lui fallut désormais entretenir et cultiver. Propriétaire du château dont il ne restera plus qu'une "grosse ferme " (Les Géorgiques, p. 144) lorsque Claude Simon visitera la propriété, il put ainsi devenir Lacombe de Saint-Michel. Son fils aîné, Jean Lacombe (1694-1759), eut à son tour trois fils, dont l'aîné, Eugène (1720-1795), deuxième du nom, eut deux fils et deux filles, l'aîné des fils, Jean-Pierre (5 mars 1753-1812), abandonnant la particule sous la Révolution pour devenir JeanPierre Lacombe Saint-Michel, député à la Législative puis secrétaire de la Convention, membre du Comité de salut public et du Conseil des Anciens, général de l'armée du Nord et ambassadeur à Naples, en bref, l'ancêtre des Géorgiques qui n'oubliera pas les membres de sa famille lorsqu'ils auront choisi le camp des révolutionnaires, sa protection s'étendant au beau-frère de son unique cousine germaine, Marie-Brigitte Lacombe.

Marie-Brigitte Lacombe de Blanchefort qui avait épousé, le 4 février 1777, Jean Balssa de la Nougayrié, alors brassier au hameau du Vergnet, frère cadet de Bernard-François Balssa, le père de Balzac. Mais ce n'est qu'au début des années 1790, semble-t-il, que Bernard-

22. Honoré de Balzac, Une fille d'Eve (1834). 
François Balzac fait la connaissance de Jean-Pierre Lacombe SaintMichel, ce dernier lui faisant connaître ses amis du "Toul Artillerie " ( $G$, p. 369), notamment Choderlos de Laclos. Et si cette rencontre fut importante pour les deux hommes, elle semble l'être bien plus encore pour le géniteur d'Honoré, Lacombe Saint-Michel orientant la carrière de son protégé, conseiller du Roi avant qu'il soit nommé, le $1^{\text {er }}$ mai 1792 , trésorier au bureau central des fourrages de l'armée du Nord où il fera toute sa carrière jusqu'à sa retraite en 1819.

"Le jacobin Balzac, homme honnête et doux ", comme le décrit Restif de la Bretonne, est ainsi entré dans les subsistances militaires grâce à l'appui de son ami méridional, et c'est cette nouvelle carrière qui va lui permettre de connaître son collègue Sallambier et d'épouser sa fille Laure, alors âgée de 18 ans. Sans L.S.M., pas d'Honoré de Balzac, insiste Jean-Louis Déga, auteur de La vie prodigieuse de Bernard-François Balssa ${ }^{23}$, qui constate que si le père de Balzac connaissait le tout Paris, c'était en grande partie grâce à l'arrière-grand-père de Claude Simon. Ce que n'est pas sans ignorer le fils, même si le nom de Lacombe Saint-Michel est totalement absent de son œuvre et de sa correspondance, ce nom n'apparaissant nulle part pour la simple raison qu'il était celui d'un régicide quand le père de Balzac, jacobin acharné le jour, était sans doute, tout comme Sanson bourreau de Louis XVI, royaliste de cœur la nuit.

Lexistence du véritable Eugène de Rastignac que fut le père de Balzac ${ }^{24}$, - qui s'éleva à la hauteur d'où les hommes profonds savent voir l'avenir en jugeant le passé, dira à peu près son fils -, traversant sans encombre la Révolution, l'Empire et la Restauration ayant connu tous les régimes politiques de Louis $\mathrm{XV}$ à Charles $\mathrm{X}$ et ayant su, sous chacun d'eux, se faire une place par l'utilisation de puissants réseaux de protection, est donc intimement liée à l'histoire familiale de Claude Simon. Et d'ailleurs, les réminiscences méridionales dans

23. Jean-Louis Déga, auteur de La vie prodigieuse de Bernard-François Balssa (Père d'Honoré de Balzac), Aux sources historiques de la Comédie humaine. Editions Subervie, Rodez, 1998.

24. Bernard-François Balzac mourra à Paris en 1829, l'année où son fils Honoré, à trente ans, signera pour la première fois sous son vrai nom le premier roman de La Comédie Humaine: Les Chouans. 
l'œuvre de Balzac sont essentiellement liées à l'influence de ce père, notamment au niveau de l'onomastique qui renvoie à la généalogie dont le romancier s'est fait le spécialiste. Ce qu'est aussi, après tout, et Les Géorgiques nous en donne la preuve, Claude Simon, comme si l'obsession généalogique ${ }^{25}$ de Balzac, et sa volonté de faire concurrence à l'état-civil avec des milliers de personnages fictifs, ou prétendus tels, et qui s'explique par l'existence de secrets de famille soigneusement dissimulés (emprisonnement du père, exécution de l'oncle, naissance adultérine du frère...), était une obsession partagée par tous les écrivains de la famille.

D'autant que si l'histoire familiale informe l'œuvre de Balzac, cette histoire est aussi celle des Lacombe. Le héros de la Révolution qui a tué son frère Jean-Marie-Eugène, le régicide cachant un fratricide, sont deux thèmes qui obséderont aussi bien Simon que Balzac $^{26}$. Nul doute, en effet, que le drame familial chez les Lacombe Saint-Michel, qui eut un certain retentissement au niveau national,

25. Balzac est l'écrivain généalogique par excellence. Et l'importance de son père et de ses fréquentations, dans son œuvre, est évidente. Sa volonté de faire concurrence à l'état civil peut s'expliquer par la nécessité de se recomposer un environnement familial, l'affaire Balssa l'ayant à tout jamais coupé de sa famille tarnaise. Affaires de famille auxquelles L.S.M. était intimement lié. Les personnages réapparaissent ainsi d'un roman à l'autre, principe sur lequel repose $\mathrm{La} C o$ médie humaine, Jacques Ameil parlant même de comédie généalogique. Et bien que l'ambition de Claude Simon ne soit pas, comme celle de Balzac, de peindre « toute la société ", la généalogie est importante dans son projet.

26. Et s'il arrive un moment, dans la vie intérieure des familles, où les enfants deviennent, soit volontairement, soit involontairement, les juges de leurs parents, il arrive aussi un moment où les frères se retournent l'un contre l'autre. Il faut battre le frère quand il est chaud! Son frère cadet, Jean-Marie-Eugène, était royaliste et avait émigré. Il était né en 1754 à Saint-Michel de Vax et était capitaine d'artillerie à 45 ans quand il émigra au moment de la Révolution à Pointe-à-Pitre. Mais il était revenu clandestinement en 1798 pour animer un centre de chouannerie du Nord Aveyron du côté de Montsalvy sur les bords du Lot. C'est en revenant de voir sa famille à Saint-Michel de Vax qu'il fut arrêté à Espinas près de Caylus. Il fut conduit devant la commission militaire siégeant à Périgueux qui le 29 mai 1799 le condamna à mort en application des lois votées par son frère. Il fut fusillé à Périgueux le même jour. Jean-Pierre avait écrit au Préfet de la Charente-Maritime qu'il avait toutes raisons de croire que son frère avait été tué à l'armée du Rhin et que le prisonnier ne pouvait être qu'un imposteur. 
et qui empêcha certainement L.S.M., comme l'explique Claude Simon à la fin des Géorgiques, d'accéder au ministère des Relations extérieures, ait inspiré l'auteur de La Comédie humaine. Les thèmes $\mathrm{du}$ régicide et $\mathrm{du}$ fratricide qui hantent plusieurs romans doivent d'autant plus au souvenir des Lacombe Saint-Michel que les faits étaient connus non seulement de Jean Balssa, dont l'épouse, on l'a dit, était la cousine germaine des deux frères Lacombe Saint-Michel, mais aussi de son ami Bernard-François Balzac, frère de Jean Balssa et père d'Honoré. Ainsi, dans Mémoires de deux jeunes mariées et dans Illusions perdues, le comte de Maucombe rappelle la situation des frères Lacombe, l'un faisant voter un décret au nom duquel l'autre sera, le 29 mars 1799, soit neuf jours après la naissance d'Honoré de Balzac, passé par les armes. Jean-Pierre Lacombe Saint-Michel restera ainsi considéré dans sa famille, demeurée en majorité royaliste, d'où les relations difficiles qu'il entretient avec sa mère et que décrit Claude Simon, comme un fratricide. Et on pourrait en dire encore beaucoup sur l'impact négatif, dans le contexte politique de la Terreur blanche, qu'eut l'ancêtre de Claude Simon lors de l'affaire Balssa, affaire criminelle qui entraîna la condamnation d'un innocent, l'oncle d'Honoré de Balzac, et qui orientera l'œuvre et la vie de ce dernier, qui avait vingt ans lorsque Louis Balssa fut exécuté. Cette même année, le neveu abandonnait sa carrière juridique pour celle des Lettres et publiait son premier ouvrage littéraire, la tragédie Cromwell, ne se lançant alors dans son œuvre surhumaine que pour se libérer, semble-t-il, des fantômes familiaux issus des placards de son père mais aussi de ceux de son protecteur qui inspirera, cent cinquante ans plus tard, un nouveau romancier...

"Rien ne forme l'âme comme une dissimulation constante au sein de la famille ", écrivait Balzac dans Une ténébreuse affaire. Pas plus autobiographique, dès lors, que l'œuvre de Balzac dont l'état civil n'est pas plus imaginaire que celui de Claude Simon dans Les Géorgiques. De même qu'on ne peut lire l'œuvre de Balzac fils sans lire entre les lignes l'histoire de Balzac père, on ne peut pas lire Les Géorgiques sans apprécier le rapport problématique de Claude Simon à l'œuvre de son illustre aîné. Dans la Chronologie de La Pléiade, à l'année 1941 est écrit : "Simon lit beaucoup : Conrad, Dostoïevski, Tchekhov, tout Proust [...], l'œuvre complète de Balzac. » Qu'il lit 
en effet, à la dernière page de L'Acacia : "Chez un bouquiniste, il acheta les quinze ou vingt tomes de La Comédie humaine reliés d'un maroquin brun-rouge qu'il lut patiemment, sans plaisir, l'un après l'autre, sans en omettre un seul "(L'Acacia, p. 379) ${ }^{27}$. Lisant Balzac comme il relirait des brouillons ou des épreuves, autrement dit, sans plaisir, puisque la quête asymptotique de la perfection exige que l'on ne se satisfasse jamais complètement ce que l'on a écrit, ou comme il relirait les archives de son aïeul, sans plaisir, mais en sachant bien, et c'est la dernière ligne de L'Acacia, que c'est seulement après avoir lu tout Balzac qu'il pourra lui-même se mettre à l'écriture.

Et c'est encore Balzac qu'il cite dans son Discours de Stockholm, et par deux fois, Balzac " hardiment novateur ", Balzac qu'il veut tirer d'affaire, s'en prenant à ceux, " épigones attardés qui, un siècle et demi plus tard, le proposent en exemple ${ }^{28}$ " " du roman traditionnel $^{29}$ " à rejeter, ajoutant ensuite : "Avec Balzac (et c'est là peut-être que réside son génie), on voit apparaître de longues et minutieuses descriptions de lieux ou de personnages, descriptions qui au cours du siècle se feront non seulement de plus en plus nombreuses mais, au lieu d'être confinées au commencement du récit ou à l'apparition des personnages, vont se fractionner, se mêler à des doses plus ou moins massives au récit de l'action, au point qu'à la fin elles vont jouer le rôle d'une sorte de cheval de Troie et expulser tout simplement la fable à laquelle elles étaient censées donner $\operatorname{corps}^{30}$. " En d'autres termes, selon Claude Simon, sans Balzac, pas de Proust. Et, a fortiori, si on le prend au mot, pas de Claude Simon. Mais sans Lacombe Saint-Michel, pas de Balzac.

$\mathrm{Ou}$, pour le dire autrement, et pour le dire par le détour de l'anecdote, sans la canne de L.S.M., cette canne, rappelons-le, qu'il a la main lorsqu'il est terrassé par une attaque d'apoplexie à l'âge de cinquante-neuf ans, pas de "canne aux turquoises", cette canne que

27. Rappelons seulement que la couleur "brun-rouge " rappelle inévitablement la couleur de l' "encre brune, couleur rouille " $(G$, p. 377) des innombrables lettres envoyées à Batti par L.S.M.

28. Claude Simon, Euvres, op. cit., p. 893.

29. Ibid., p. LXIII.

30. Id., p. 895. 
Balzac, le 18 août 1834, commande à l'orfevre et joaillier parisien Lecointe, canne dont il parlera, dès le 30 mars 1835, soit quinze jours avant d'en régler la facture, à Mme Hanska, lui racontant qu'elle a "plus de succès en France que toutes [ses] œuvres ". Il vient alors d'imprimer à son œuvre un tournant décisif en appliquant, dans Le Père Goriot, l'idée des personnages qui reparaissent d'un roman à l'autre. Malgré le succès du roman, il est en butte à la critique. Il lui faut convaincre, briller de mille feux, être un homme en vue pour être lu. Avec sa canne de dandy, il ne passe pas inaperçu. Sa canne, d'ailleurs, fait couler beaucoup d'encre, déchaîne la verve des caricaturistes et devient un «bijou européen » dont l'éclat rejaillit sur Balzac. Il la conservera jusqu'à sa mort, survenue en 1850, un 18 août, le jour même où il l'avait commandée seize ans plus tôt...

La boucle est bouclée, pour ainsi dire. De Lacombe Saint-Michel à Rastignac, en effet, telle serait l'ampleur de la " démesure ${ }^{31}$ "! D'un Eugène à l'autre, en l'occurrence. Du jeune étudiant de la pension Vauquer à Claude Eugène Simon, fils d'Antoine Eugène Simon, neveu de Marie et Eugénie. Du jeune ambitieux logeant rue NeuveSainte-Geneviève, qui était, du temps de François ${ }^{\text {er }}$, réputée pour ses maisons de jeu dont le fameux Tripot des 11.000 Diables, à, du côté maternel cette fois, Eugène Lacombe, premier et deuxième du nom, puis "Jean-Marie-Eugène L.S.M. » $(G$, p. 441), frère de JeanPierre dont le fils, son héritier universel, s'appellera, en toute logique, "Eugène-Maurice-Henri » ( $G$, p. 400). En toute logique, puisque les prénoms, comme les bons mots, ont l'art de se transmettre de génération en génération. Mais aussi parce qu'Eugène de Rastignac - "A nous deux maintenant ! " - est à la fois l'incarnation du père de Balzac monté à Paris pour y faire fortune, mais aussi le modèle suivi par Claude Simon qui fera exactement le même voyage, de la vallée du Viaur dans le Tarn à la capitale, pour y trouver la fortune littéraire. Eugenos signifie, en grec, bien né, de noble race. Comme si ce prénom, dont on sait la popularité au XIX ${ }^{\mathrm{e}}$ siècle, avait ce pouvoir de relier aussi bien les Simon aux Lacombe que les Lacombe aux Balzac puisque de toute évidence, comme l'explique Claude Simon dans son Discours de Stockholm, "l'art s'autogénère pour ainsi dire 
par imitation de lui-même ${ }^{32}$ ". Ainsi la transmission des caractères héréditaires entre les géniteurs et leur descendance en passe-t-elle par ce prénom qui réunit les deux extrêmes de la littérature française. Et lorsqu'il est question de la place des Géorgiques dans l'œuvre de Claude Simon, c'est aussi et avant tout de la place de ce roman dans la littérature française dont il est question.

C'est que malgré les flous, les doutes, les secrets, les mensonges, les incestes, les adultères et Dieu sait quoi encore, le fils de "l'arrière-petite-fille du général d'Empire " (L'Acacia, p. 127), se glissant dans la chambre matrimoniale, assistant à l'acte sexuel et à sa propre conception $^{33}$, réinvente la scène primitive. Mais cette scène primitive n'en reste pas moins boiteuse. Car la guerre fait mal et lorsqu'elle ne tue pas, comme elle a tué le père de Claude Simon, elle blesse, elle handicape et fait boiter les hommes : "Il est blessé à la jambe à Farinole. » ( $G$, p. 22) Celui qui est blessé au genou, pour être exact, et comment ne pas l'être, Les Géorgiques y revenant sans cesse, c'est L.S.M. ${ }^{34}$ : "A Stralsund sa vieille blessure à la jambe lui occasionne des grandes douleurs lorsqu'il doit rester trop longtemps à cheval. " ( $G$, p. 29) Plus tard, "boitant peut-être encore du coup de feu reçu à Saint-Florent " $(G$, p. 384), "il écrit une diatribe contre Masséna dont les fourgons sont la fable de l'armée et qui a eu l'indignité de ne prêter que 25 louis à Soult resté sur le champ de bataille avec une jambe cassée. » $(G, 28)$ Le récit mélange bientôt les époques et le narrateur (ou Georges) se souvient alors de la séquence de la traversée du village de Sars-Poteries en mai 1940, et de «l'ordonnance [à] la cuisse éraflée par une balle » $(G$, p. 53). D'autant que le JeanPierre boiteux, c'est aussi, dans Le Tramway, le Georges traînant der-

32. "Mais il serait encore nécessaire de préciser la nature de cette imitation, car l'art s'autogénère pour ainsi dire par imitation de lui-même : de même que ce n'est pas le désir de reproduire la nature qui fait la peinture mais la fascination du musée, de même c'est le désir d'écrire suscité par la fascination de la chose écrite qui fait l'écrivain, la nature se bornant pour sa part, comme le disait spirituellement Oscar Wilde, à 'imiter l'art'... ", Discours de Stockholm (CEuvres, p. 891).

33. Cf. L'Acacia, p. 145 et Histoire, p. 401-402.

34. C'est à cette occasion, d'ailleurs, qu'il rencontre Napoléon Bonaparte, alors jeune capitaine d'artillerie, et qu'il contribue à le faire évacuer de Corse avec sa famille quand les choses tournent mal. 
rière lui " sa douloureuse et lourde jambe à la rotule broyée qu'il avait ramenée de la guerre " (Le Tramway, p. 37), ou celui qui, dans La Route des Flandres, faisait déjà des «rêves boiteux » (La Route des Flandres, p. 169).

Et que depuis, si le héros boite, c'est parce que tout boite ${ }^{35}$. Jean-Pierre Lacombe Saint-Michel boite ${ }^{36}$, et c'est toute la famille qui, clopin clopant, de page en page, et d'un roman à l'autre, cherche son équilibre. Georges veut être un " héros » (G, p. 374), " poursuivant à coups de baïonnette une silhouette d'homme à moitié vêtu " ( $G$, p. 209), mais il laisse finalement échapper l'ennemi à trop boiter sur le remblai. Ce qui lui évite de commettre l'irréparable. Car ce qui boite, c'est cette mécanique romanesque à la fois blessée et indispensable, indispensable mais comme peut l'être une "vieille domestique boiteuse » ( $G$, p. 172), indispensable comme «l'interne logique de la matière [et] ses implacables mécanismes » ( $G$, p. 352), et "claudicante " $(G$, p. 192) parce qu'on se trouve alors, à l'endroit précis et à l'heure précise, sur " la trajectoire [d'une] balle " $(G, \mathrm{p}$. 361), et d'une balle qui fait hasard, d'une balle qui fait le hasard de l'écriture, puisqu'il n'y en a pas d'autre pour Claude Simon, et qu'écrire, pour lui, c'est un peu comme faire la guerre, la guerre pour la guerre comme, disait-il, "l'art pour l'art ", mais que c'est aussi, de fait, en porter la blessure, la marque, ainsi que la révolte, la même révolte qui, siècle après siècle, des Lacombe Saint-Michel aux Balzac, s'est transmise de Georges à Claude, Claude Simon dont le prénom même claudique.

35. Voir Aymeric Glacet, op. cit., p. 85-113.

36. " $1^{\circ}$ à la partie inférieure et antérieure de la jambe gauche une cicatrice large, violette et mal consolidée qui se rouvre à la moindre fatigue malgré l'application d'un bandage roulé auquel il s'est assujetti, $2^{\circ}$ une autre cicatrice, mais un peu plus solide, à la partie inférieure de la face interne de la cuisse du même côté ; ces deux cicatrices sont le résultat de deux coups de feu » $(G$, p. 66). 